\title{
CIPHI National Annual General Meetings and Conference Locations
}

\section{Tim Roark}

\section{National Historian CIPHI}

Tim Roark, CIPHI National Historian, has gathered a list of the CIPHI National Annual Meetings and Conference locations since the inception of CIPHI. Please see below for the year and number of the Conference, and the location.

No Annual Meetings were held in 1915 (World War I) and 1945 (World War II). Manitoba has held the most Conferences with 28, followed by Ontario with 23, and British Columbia with 14. This is followed by Alberta (13), Saskatchewan (12), Quebec (4), Nova Scotia (4), Newfoundland (2), and Prince Edward Island (1). One was held in the US (Chicago) in 1996.

Cities that have hosted most frequently include Winnipeg (27) plus the Inaugural meeting, Vancouver (9), Edmonton (7), Regina (4), Saskatoon (5), Toronto (4), Banff (3), Halifax (4), London (3), Montreal (3), Ottawa (4), Calgary (2), Niagara (2), Richmond (2), St. John (2), Victoria (2), Waskesiu (2), and Windsor (2). Thirteen cities have hosted one time each.

\author{
2018 84th Saskatoon, SK \\ 2017 83rd Richmond, BC \\ 2016 82nd Edmonton, $\mathrm{AB}$ \\ 2015 81st Ottawa, ON \\ 2014 80th St. Johns, NL \\ 2013 79th Winnipeg, MB (100 Years) \\ 2012 78th Blue Mountains, ON \\ 2011 77th Halifax, NS \\ 2010 76th Vancouver, BC \\ 2009 75th Kananaskis, AB \\ 2008 74th St. Johns, NL \\ 2007 73rd Kelowna, BC \\ 2006 72nd Regina, SK \\ 2005 71st Toronto, ON \\ 2004 70th Charlottetown, PEI \\ 2003 69th Edmonton, AB \\ 2002 68th Fredericton, NB \\ 2001 67th Winnipeg, MB \\ 2000 66th Vancouver, BC \\ 1999 65th Saskatoon, SK \\ 1998 64th Ottawa, ON \\ 1997 63rd Banff, AB \\ 1996 62nd Chicago, IL \\ 1995 61st Victoria, BC \\ 1994 60th Halifax, NS \\ 1993 59th London, ON \\ 1992 58th Winnipeg, MB \\ 1991 57th Edmonton, AB \\ 1990 56th Thunder Bay, ON \\ 1989 55th Toronto, ON \\ 1988 54th Vancouver, BC \\ 1987 53rd Saint John, NB \\ 1986 52nd Niagara Falls, ON \\ 1985 51st Saskatoon, SK \\ 1984 50th Banff, AB \\ 1983 49th North Bay, ON \\ 1982 48th Victoria, BC
}

\begin{tabular}{|c|c|c|}
\hline 1981 & 47 th & Winnipeg, MB \\
\hline 1980 & 46th & Saskatoon, SK \\
\hline 1979 & 45 th & Banff, AB \\
\hline 1978 & 44th & Cambridge, ON \\
\hline 1977 & $43 \mathrm{rd}$ & Halifax, NS \\
\hline 1976 & $42 \mathrm{nd}$ & Richmond, BC \\
\hline 1975 & $41 s t$ & Granby, QC \\
\hline 1974 & 40th & Edmonton, $\mathrm{AB}$ \\
\hline 1973 & 39th & Winnipeg, MB \\
\hline 1972 & 38th & Windsor, ON \\
\hline 1971 & 37 th & Regina, SK \\
\hline 1970 & 36th & St. John, NB \\
\hline 1969 & 35th & Vancouver, BC \\
\hline 1968 & 34 th & Calgary, AB \\
\hline 1967 & $33 \mathrm{rd}$ & Montreal, QC \\
\hline 1966 & $32 \mathrm{nd}$ & London, ON \\
\hline 1965 & $31 s t$ & Winnipeg, MB \\
\hline 1964 & 30th & Halifax, NS \\
\hline 1963 & 29th & Vancouver, BC \\
\hline 1962 & 28th & Waskesiu, SK \\
\hline 1961 & 27 th & Edmonton, AB \\
\hline 1960 & 26th & Montreal, QC \\
\hline 1959 & 25 th & Niagara Falls, ON \\
\hline 1958 & 24th & Windsor, ON \\
\hline 1957 & $23 \mathrm{rd}$ & Vancouver, BC \\
\hline 1956 & $22 \mathrm{nd}$ & Winnipeg, MB \\
\hline 1955 & $21 s t$ & Waskesiu, SK \\
\hline 1954 & 20th & Kingston, ON \\
\hline 1953 & 19th & Vancouver, BC \\
\hline 1952 & 18th & Sudbury, ON \\
\hline 1951 & 17 th & Edmonton, $\mathrm{AB}$ \\
\hline 1950 & 16th & Montreal, QC \\
\hline 1949 & 15 th & Ottawa, ON \\
\hline 1948 & 14 th & Vancouver, BC \\
\hline 1947 & 13 th & Winnipeg, MB \\
\hline 1946 & 12 th & Toronto, ON \\
\hline 1945 & & \\
\hline
\end{tabular}

1944 11th Winnipeg, MB
1943 10th Winnipeg, MB
1942 9th Winnipeg, MB
1941 8th Winnipeg, MB
1940 7th Winnipeg, MB
1939 6th Winnipeg, MB
1938 5th Winnipeg, MB
1937 4th Winnipeg, MB
1936 3rd Winnipeg, MB
1935 2nd Winnipeg, MB
1934 1st Winnipeg, MB
1933 * Winnipeg, MB
1932 Winnipeg, MB
1931 London, ON
1930 Winnipeg, MB
1929 Moose Jaw, SK
1928 Vancouver, BC
1927 Toronto, ON
1926 Brantford, ON
1925 Winnipeg, MB
1924 Fort William, ON
1923 Calgary, AB
1922 Winnipeg, MB
1921 Ottawa, ON
1920 Edmonton, AB
1919 Saskatoon, SK
1918 Winnipeg, MB
1917 Regina, SK
1916 Winnipeg, MB
1915 FOTES:
1914 Winnipeg, MB
1913 Regina, SK
1913 Winnipeg, MB (Inaugural Mtg.)
*Years pre-national incorporation

